\title{
On the interference between the two microphones in free-field reciprocity calibration
}

\author{
Barrera Figueroa, Salvador; Rasmussen, Knud; Jacobsen, Finn; Muñíc, Lorenzo
}

Published in:

Acoustical Society of America. Journal

Link to article, DOI:

$10.1121 / 1.1795333$

Publication date:

2004

Document Version

Publisher's PDF, also known as Version of record

Link back to DTU Orbit

Citation (APA):

Barrera Figueroa, S., Rasmussen, K., Jacobsen, F., \& Muñíc, L. (2004). On the interference between the two microphones in free-field reciprocity calibration. Acoustical Society of America. Journal, 116(5), 2771-2778. https://doi.org/10.1121/1.1795333

\section{General rights}

Copyright and moral rights for the publications made accessible in the public portal are retained by the authors and/or other copyright owners and it is a condition of accessing publications that users recognise and abide by the legal requirements associated with these rights.

- Users may download and print one copy of any publication from the public portal for the purpose of private study or research.

- You may not further distribute the material or use it for any profit-making activity or commercial gain

- You may freely distribute the URL identifying the publication in the public portal

If you believe that this document breaches copyright please contact us providing details, and we will remove access to the work immediately and investigate your claim 


\title{
On the interference between the two microphones in free-field reciprocity calibration
}

\author{
Salvador Barrera-Figueroa, ${ }^{\text {a) }}$ Knud Rasmussen, ${ }^{\text {b) }}$ and Finn Jacobsen ${ }^{\mathrm{c})}$ \\ Acoustic Technology, Ørsted·DTU, Technical University of Denmark, Building 352, DK-2800, \\ Kgs. Lyngby, Denmark \\ Lorenzo Muñíz ${ }^{d)}$ \\ Centro Nacional de Metrología, División de Acústica y Vibraciones, Carretera a Los Cués km 4.5, \\ El Marqués, Querétaro, México
}

(Received 19 January 2004; revised 12 May 2004; accepted 30 July 2004)

\begin{abstract}
One of the fundamental assumptions in free-field reciprocity calibration of microphones is that the microphones can be substituted by point sources at the positions where the acoustic centers are located. However, in practice the microphones have finite dimensions and, at the distance and in the frequency range where the measurements are made, the direct wave and the subsequent reflections from the microphones interfere with each other, creating a "standing wave." This interference effect gives rise to deviations from the inverse distance law, indicating that the free-field assumption is not strictly valid. The interference has been thought to be caused by specular reflection between the parallel diaphragms of the microphones, and a solution based on tilting the axis of one of the microphones a few degrees has been proposed, but never examined in practice. In this paper a time-selective technique is applied for analyzing the interference and for removing it in the time domain. It is shown that the phenomenon is due to multiple backscattering rather than specular reflection. Thus tilting one of the microphones does not alleviate the problem, as also demonstrated experimentally. However, the time-selective technique is quite effective in removing the interference effect and other disturbances from the direct wave between the microphones. (C) 2004 Acoustical Society of America. [DOI: 10.1121/1.1795333]
\end{abstract}

PACS numbers: 43.20.Fn, 43.20.Ye, 43.38.Kb [AJZ]

Pages: 2771-2778

\section{INTRODUCTION}

Free-field reciprocity calibration of condenser microphones is a well-established technique used for obtaining the absolute sensitivity of condenser microphones under freefield conditions. This is reflected in the fact that there is an international standard for such calibrations. ${ }^{1}$ The theoretical foundation of the free-field reciprocity calibration of microphones was developed by MacLean ${ }^{2}$ and Wathen-Dunn. ${ }^{3}$ Descriptions of the practical implementation of the technique have been given, e.g., by Rudnick and Stein, ${ }^{4}$ and more recently by researchers from a number of national metrology laboratories. ${ }^{5-7}$ Some years ago an international comparison among several national metrology laboratories was carried out, ${ }^{8}$ yielding results that have motivated further research on certain aspects of the calibration technique.

One of the basic assumptions of the underlying theory is that the microphones can be substituted by point sources/ receivers with a given diffraction factor. ${ }^{1-3}$ This greatly simplifies the procedure, and a quantity proportional to the product of the free-field sensitivities of the two microphones, the electrical transfer impedance, can be obtained. ${ }^{1}$ The diffraction factor is the result of the finite size of the microphones. ${ }^{9,10}$ However, another result of the finite size of the microphones, a phenomenon often referred to as a

\footnotetext{
${ }^{a)}$ Electronic mail: sbf@oersted.dtu.dk

${ }^{b)}$ Electronic mail: kr@oersted.dtu.dk

${ }^{c}$ Electronic mail: fja@oersted.dtu.dk

d)Electronic mail: 1muniz@cenam.mx
}

"standing wave" between the microphones, has been observed in some practical implementations of the technique. ${ }^{3-5,8}$ This problem has been thought to be caused by "specular" reflections between the parallel diaphragms of the microphones. Since such an interference represents a deviation from the basic assumptions of the technique, it has a negative effect on the accuracy of the calibration.

One of the solutions proposed for solving this problem consists in finding the "optimal" distance where the magnitude of the "standing wave" becomes negligible. ${ }^{3}$ However, as the distance between the microphones is increased, the influence of reflections from the walls of the (in practice less than perfect) anechoic chamber will be more serious, making this solution impractical. It has also been suggested to tilt the axis of one of the microphones a few degrees, ${ }^{4,5}$ but this method has never been examined in practice because of the practical difficulties in the mechanical positioning of the microphones.

The interference problem has hitherto been analyzed in the frequency domain by comparing transfer impedances measured using different distances between the microphones. ${ }^{5,8}$ However, a fast Fourier transform (FFT)-based, time-selective technique has recently been developed and applied to the free-field reciprocity calibration. ${ }^{11}$ This technique makes it possible to determine the impulse response corresponding to the electrical transfer impedance and thus identify the direct impulse response between the microphones and responses resulting from unwanted reflections. Subse- 
quently the undesirable components can be eliminated by means of time-windowing.

Quantifying the interference phenomenon is not easy. There is no analytical solution to the wave equation for the geometry of the two microphones facing each other. Numerical methods such as the boundary element method (BEM) can solve the problem, but substantial computer resources will be required. In a recent paper concerned with the scattering of an array of closely spaced microphones, Ihlárova and Jacobsen used a three-dimensional BEM formulation for simulating a $3 \times 3$ microphone array and added the pressure increase on the diaphragm of the microphone located in the center of the array caused by each of the surrounding microphones, one at a time. ${ }^{12}$ Although this procedure does not give the complex interference that occurs when all the microphones are interacting at the same time, it gives a fairly good approximation. This approximate procedure was developed because of the large amount of computational resources required for direct modeling of the microphone array. Although there are only two microphones in the free-field reciprocity arrangement, it is specified in the standard that the length of the rods on which the microphones are mounted should be "long compared to the diameter of the microphones," to avoid interference from the end of the rods, ${ }^{1}$ and in practice rods more than 20 times longer than the diameter are used. Thus, the number of required elements of the BEM model would be as prohibitive as in case of the microphone array studied in Ref. 12. However, the case where the axes of the microphones are aligned can be modeled using a far more economical axisymmetric formulation. ${ }^{13}$

The problem of the interference between the transmitter microphone and the receiver microphone is not only relevant in a free-field reciprocity calibration, but also for comparison methods where the transducers under study are closely located in front of each other. Thus, our purpose in this paper is to analyze the interference between the source and the receiver in free-field calibration in general. The time-selective technique described in Ref. 11 is used for examining the "standing wave" when the microphones are aligned and for investigating the effect of tilting one of the microphones. The experiments are supplemented by BEM calculations.

\section{THE INTERFERENCE MECHANISM}

Consider a source with finite dimensions and another (passive) body, the receiver, some distance away in a free field. If a portion of the surface of the source moves with a given velocity distribution it will generate a sound wave that propagates away from the source. A fraction of the sound energy is scattered by the receiver back toward the source. When this backscattered wave impinges on the body of the source, it gives rise to yet another wave that propagates toward the receiver. Thus, the receiver will register the direct wave and the secondary reflection from the source.

In the case at hand the source and the receiver are condenser microphones in a free-field reciprocity calibration setup. As specified in the standard, the two microphones are mounted on long cylindrical rods with the same diameter and placed in front of each other in an anechoic room. ${ }^{1}$ It would be interesting to estimate the ratio of the direct wave to the secondary reflection at the receiver microphone. To get an idea about this ratio three cases can be considered: pure specular reflection, scattering when the dimensions of the two bodies are much shorter than the wavelength, and scattering when the dimensions are much larger than the wavelength.

\section{A. Specular reflection}

Consider a point source located on an infinite, rigid baffle, and a second parallel, rigid baffle located at a distance $d$ in front of the baffled source. The receiver is the point on the second baffle directly in front of the point source. A wave emitted by the source will travel the distance $d$ toward the baffled receiver, where it will be reflected back. The reflected wave can be regarded as generated by an image source located at a distance $d$ behind the reflecting baffle. The reflected wave will travel back to the baffle with the original source, where it is reflected once again. This second reflection can be thought to be caused by a second image source located at a distance of $2 d$ behind the baffled source. Thus, when the second reflection reaches the receiver in front of the source, the sound wave has traveled three times the distance between the baffles. Note that the second image source has the same strength as the original source, because the energy is fully reflected from the infinite baffles. Therefore, the logarithmic ratio of the amplitude of the second reflection to the amplitude of the direct wave at the receiver position is

$$
R=20 \log \left(\frac{d}{3 d}\right)=-9.54 \mathrm{~dB} .
$$

The relation between the amplitudes of reflected waves at two different distances between the baffles can be expressed as the logarithmic ratio of the distances,

$$
R^{\prime}=20 \log \left(\frac{d_{1}}{d_{2}}\right) \text {. }
$$

\section{B. Scattering}

When the diameters of the source and the receiver are smaller than or comparable with the wavelength, specular reflection is not very realistic; it seems more reasonable to assume that the sound wave generated by the source is scattered by the second body. This means that the reflected energy will only be a fraction of the energy emitted by the source. This fraction may be expected to be related to the solid angle formed by the receiver, as seen from the source. The most adequate way of establishing the relation between the direct wave and the backscattering from the receiver microphone would be to solve the wave equation with the boundary conditions posed by the bodies of the microphones. However, this problem cannot be solved analytically. Therefore the simple case of scattering by a sphere is analyzed instead, on the assumption that it can, at least, illustrate the phenomenon and give an idea about orders of magnitude.

Assuming harmonic variation with the $e^{-j \omega t}$ sign convention, the sound pressure scattered by a solid sphere of radius $a$, centered at the origin of a spherical coordinate system, can be calculated from the expression ${ }^{14}$ 


$$
\begin{aligned}
p_{s}(r, \theta)= & -A \sum_{m=0}^{\infty}\left\{(2 m+1) j^{m+1} e^{-j \delta_{m}}\right. \\
& \left.\times \sin \delta_{m} P_{m}(\cos \theta) h_{m}(k r)\right\},
\end{aligned}
$$

where $A$ is the amplitude of a plane wave incident in the direction $\theta=0, h_{m}(x)$ is the spherical Hankel function of first kind and order $m, P_{m}$ is the Legendre function of order $m, k$ is the wave number, $r$ is the distance to the observation point, and the angle $\delta_{m}$ is defined as

$$
\delta_{m}=\arctan \left\{\frac{(m+1) j_{m+1}(k a)-m j_{m-1}(k a)}{m n_{m-1}(k a)-(m+1) n_{m+1}(k a)}\right\} .
$$

In this expression, $j_{m}$ and $n_{m}$ are the spherical Bessel and Neumann function of order $m$.

If the two transducers in a free-field calibration setup may be approximated by spheres, and if it can be assumed that the wave from one sphere may be regarded as locally plane when it arrives at the other sphere, it follows that the amplitude of the sound wave impinging on the receiving sphere after being scattered by both spheres in succession is

$$
\begin{aligned}
p_{s, 2}= & -p_{s} \sum_{m=0}^{\infty}\left\{(2 m+1) j^{m+1} e^{-j \delta_{m}}\right. \\
& \left.\times \sin \delta_{m} P_{m}(\cos \pi) h_{m}(k r)\right\} \\
= & A\left[\sum _ { m = 0 } ^ { \infty } \left\{(2 m+1) j^{m+1} e^{-j \delta_{m}}\right.\right. \\
& \left.\left.\times \sin \delta_{m} P_{m}(\cos \pi) h_{m}(k r)\right\}\right]^{2},
\end{aligned}
$$

where the distance between the spheres is $r$. It is apparent that the ratio of the secondary reflection to the incident sound pressure depends on three quantities; the radius of the spheres (which is present in the angles $\delta_{m}$ ), the distance between the spheres, and the frequency. When $k a \ll 1$ the infinite series in Eq. (5) can be truncated to the first two terms, $m=0$ and $1,{ }^{14}$ and thus

$$
\begin{aligned}
\frac{p_{s, 2}}{A} \approx & \left(j e^{-j \delta_{0}} \sin \delta_{0} P_{0}(\cos \pi) h_{0}(k r)\right. \\
& \left.-3 e^{-j \delta_{1}} \sin \delta_{1} P_{1}(\cos \pi) h_{1}(k r)\right)^{2} \\
= & \left\{\frac{e^{j k r}}{k r}\left[e^{-j \delta_{0}} \sin \delta_{0}+3 e^{-j \delta_{1}} \sin \delta_{1} \cos \pi\left(1+\frac{j}{k r}\right)\right]\right\}^{2},
\end{aligned}
$$

which, with the asymptotic values of the spherical Bessel and Neumann functions for small arguments, ${ }^{14}$ simplifies to

$$
\frac{p_{s, 2}}{A}=\left(\frac{e^{j k r}}{k r} \frac{(k a)^{3}}{3}\left(1+\frac{3}{2}\left(1+\frac{j}{k r}\right)\right)\right)^{2} .
$$

When $k a \gg 1$, Eq. (3) becomes ${ }^{14}$

$$
\left|p_{s}\right|=|A| \frac{a}{2 r},
$$

and thus

$$
\left|\frac{p_{s, 2}}{A}\right|=\left(\frac{a}{2 r}\right)^{2} \text {. }
$$

Equation (7) shows that the scattered pressure is much lower than the direct pressure at low frequencies, but depends strongly on the frequency. It can also be seen that the ratio decreases roughly by $12 \mathrm{~dB}$ as the distance $r$ is doubled. At high frequencies, Eq. (9) shows that the scattered pressure approaches a frequency-independent fraction of the direct pressure, and the ratio decreases by $12 \mathrm{~dB}$ when the distance is doubled.

Equation (3) shows that the scattered pressure depends on the cosine of the incidence angle, $\theta$, which equals $\pi$ for straight backscattering as in Eq. (6). The cosine is a slowly varying function for arguments around $\pi$. At high frequencies there is no influence of the angle at all (except at $\theta=0$ in the shadow zone behind the sphere). ${ }^{14}$ Thus, insofar as one can draw conclusions about microphones from spheres, only a modest influence of small tilting angles in the calibration setup can be expected.

It seems reasonable to present the results of the foregoing considerations in the same way as Eqs. (1) and (2). Thus, from Eq. (7), the ratio of the secondary reflection to the direct sound is

$$
R \approx 40 \log \left(\frac{5}{6} \frac{k^{2} a^{3}}{d}\right),
$$

at low frequencies, and, from Eq. (9),

$$
R=40 \log \left(\frac{a}{2 d}\right),
$$

at high frequencies, and the ratio of the amplitudes of the secondary reflection at two different distances is

$$
R^{\prime}=60 \log \left(\frac{d_{1}}{d_{2}}\right),
$$

at low as well as at high frequencies.

\section{EXPERIMENTAL RESULTS}

Some experiments have been carried out with a setup for free-field reciprocity calibration in an anechoic room using the measurement technique described in Ref. 11. The anechoic room used in these experiments was not the very small room described in Ref. 11, but DTU's "small anechoic room," with free space dimensions $4.8 \times 4.1 \times 2.9 \mathrm{~m}$ and a lower limiting frequency of about $100 \mathrm{~Hz} .{ }^{15}$ The microphones were mounted on long cylindrical rods. The electrical transfer impedance between two microphones was measured frequency by frequency, and the corresponding impulse response was calculated by an inverse fast Fourier transform. Two cases were studied, frontal incidence where the axes of the microphones coincided, and oblique incidence where one of the microphones was tilted a few degrees. The experimental arrangement is shown schematically in Fig. 1.

\section{A. Frontal incidence}

Although microphones are not spheres it is interesting to compare the experimental results with a prediction based on 

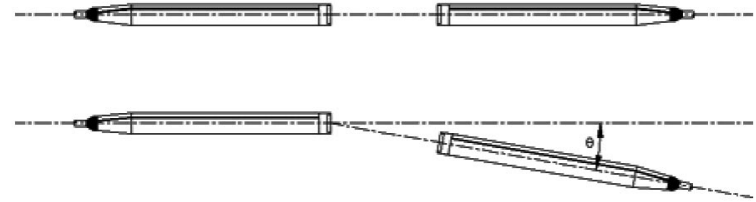

FIG. 1. A simplified sketch of the experimental arrangement. The top figure represents the case of frontal incidence, and the figure below represents the case where the axis of one of the microphones has been tilted at an angle $\theta$.

Eq. (5). The electrical transfer impedance between two microphones represents the ratio of the output voltage of the receiver microphone to the input current through the transmitter microphone. ${ }^{1}$ If microphones scatter sound as spheres then the electrical transfer impedance is modified as follows:

$$
Z_{e, 12}^{\prime}=Z_{e, 12}\left(1+\frac{p_{s, 2}}{A}\right),
$$

where $Z_{e, 12}$ is the ideal electrical transfer impedance and the second term in the parentheses represents the error due to the "standing wave." The ideal transfer impedance is calculated as described in Ref. 11 on the basis of expressions given in Ref. 1.

Figure 2(a) shows the logarithmic modulus of the Hilbert envelope of two impulse responses between two 1-in. laboratory standard (LS1) microphones of type Brüel \& Kjær (B\&K) 4160 at two different distances, 200 and $280 \mathrm{~mm}$. Figure 2(b) shows the corresponding theoretical impulse re-

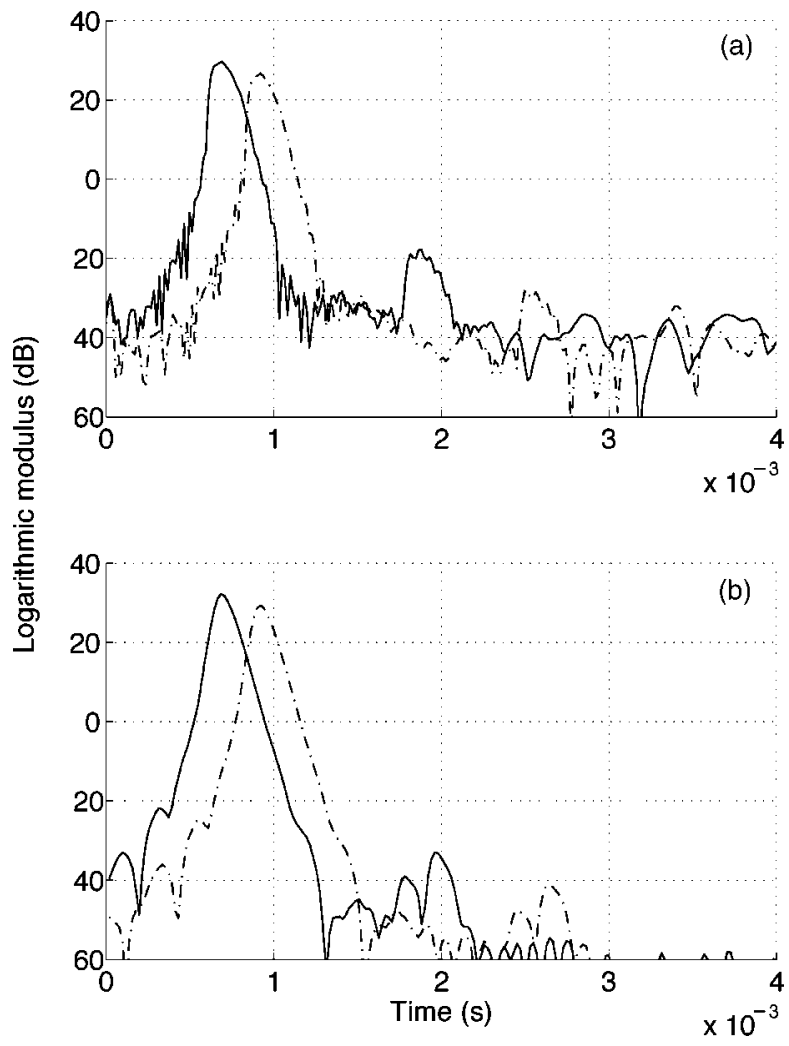

FIG. 2. The logarithmic modulus of the Hilbert envelope of the impulse response with two different distances between the microphones: - , 200 $\mathrm{mm} ;-\cdot-, 280 \mathrm{~mm}$. (a) Experimental results; (b) the calculation in which the microphones are approximated by spheres. sponses, calculated from Eq. (13), on the assumption that the microphones scatter sound as spheres with the same diameter.

Whereas it may be difficult to separate the direct sound from the reflections in the frequency response, these components are clearly differentiated in the time domain. The perturbation that occurs just after the direct impulse response is due to the "standing wave" between the microphones; as expected, it appears at an instant corresponding roughly to the time it takes a wave front to travel three times the distance between the microphones. Subsequent disturbances are caused by reflections from the walls; they appear at instants corresponding to the time it may take a wave to travel from the transmitter microphone through reflections from the walls to the receiver microphone.

According to the analysis in Sec. II the secondary reflection will decrease rapidly as the distance between the microphones is increased if the phenomenon essentially is scattering; according to Eq. (12) it should decrease by $8.8 \mathrm{~dB}$ when the distance is increased from 200 to $280 \mathrm{~mm}$. On the other hand, if the phenomenon is specular, the reflection should decrease much less, only $2.9 \mathrm{~dB}$ according Eq. (2). It can be seen from Fig. 2 that the peak value of the secondary reflection decreases by about $10 \mathrm{~dB}$ both in the experimental case and in the theoretical prediction based on approximating the microphones with spheres. This demonstrates, perhaps not surprisingly, that the phenomenon is scattering rather than specular reflection. It also confirms that the amplitude of the "standing wave" is reduced more rapidly than the direct sound when the distance between the two microphones is increased. However, this may not be a practical solution because the signal-to-noise ratio becomes very poor as the distance is increased, and reflections from the walls become more serious.

At a distance of $200 \mathrm{~mm}$ between the objects the secondary reflection is about $15 \mathrm{~dB}$ stronger compared with the direct sound in the experimental results than in the case of scattering by spheres. This is not totally unexpected since backscattering depends on the shape of the scattering object. For example, at low frequencies the amplitude of a scattered wave from a sphere differs from the scattered wave from a disk by a factor of $5 \pi / 4 .{ }^{16}$ The shape of the pulse representing the secondary reflection also depends on the scattering object; for example, the double peak seen in Fig. 2(b) is due to the fact that the sound wave travels around the sphere. Furthermore, LS1 microphones have a small front cavity or recess that may act as a resonator, increasing the backscattering; see Fig. 3.

The influence of the front cavity has been examined experimentally by measuring the electrical transfer impedance between two microphones with and without the front cavity. Figure 4 shows the Hilbert envelope of the impulse response of measurements with a pair of microphones of type B\&K 4160 and a pair of microphones of type B\&K 4145 without a protection grid located $200 \mathrm{~mm}$ from each other. The impulse responses have been normalized to the same amplitude of the direct sound.

The first disturbance after the direct impulse response is the result of the "standing wave," which appears at an in- 


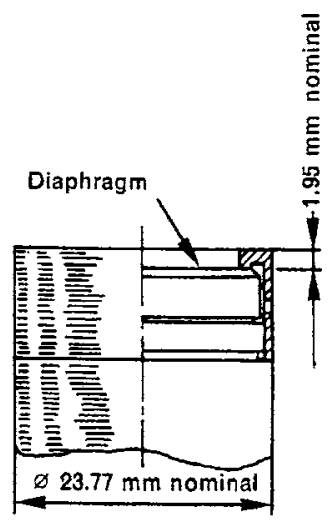

(a)

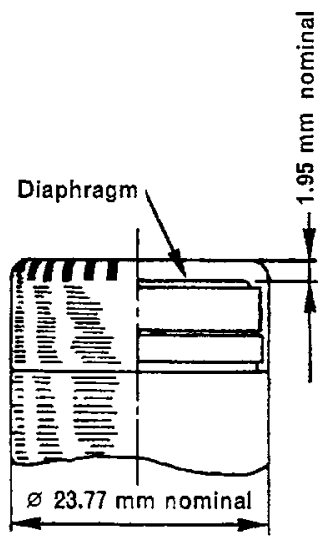

(b)

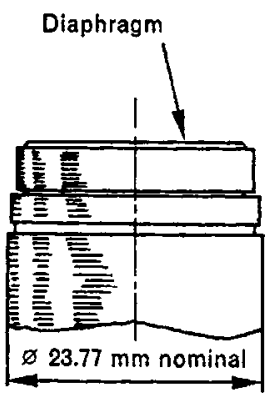

(c)
FIG. 3. (a) Microphone of type LS1 (B\&K 4160); (b) the microphone of type $\mathrm{B} \& \mathrm{~K} 4145$ with a protection grid; (c) the microphone of type B\&K 4145 without a protection grid. stant corresponding to the time it takes a wave front to travel three times the distance between the microphones. Later reflections from the mounting setup can be neglected in this analysis. It can be seen that the amplitude of the secondary reflection of the microphones with a front cavity is nearly two times larger than that of the B\&K 4145 microphones. This may be due to a transversal resonance of the front cavity, or the cavity may act as a focusing element that amplifies the backscattering.

\section{B. Oblique incidence}

Figure 5 shows the Hilbert envelope of the impulse responses between two LS1 microphones obtained from measurements where one of the microphones has been tilted $0^{\circ}$, $2^{\circ}, 4^{\circ}$, and $8^{\circ}$. The amplitudes are normalized by the amplitude of the direct sound. Again there is first an echo at about $2 \mathrm{~ms}$, which is the time it takes the secondary reflection to reach the receiver microphone. Subsequent reflections are caused by traverse rods of the mounting setup; they can be disregarded in this analysis. It can be seen that neither the amplitude nor the shape of the echo related to the "standing

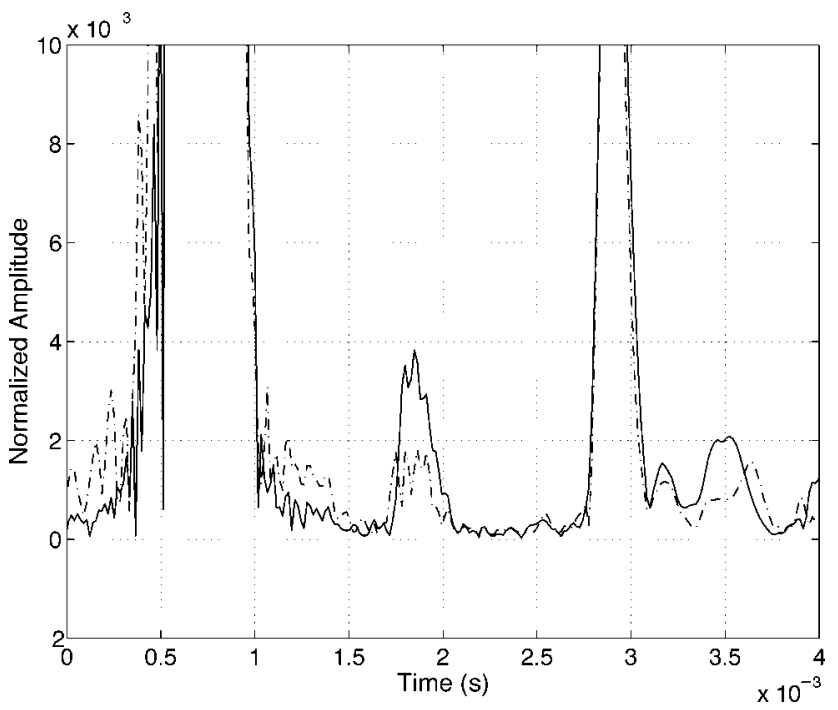

FIG. 4. A comparison between the Hilbert envelope of the normalized impulse response of the electrical transfer impedance between two microphones located at a distance of $200 \mathrm{~mm}$ from each other. -, LS1 microphones (type B\&K 4160); - - , microphones of type B\&K 4145 without a protection grid. wave" between the microphones changes significantly for tilting angles up to $4^{\circ}$, and the impulse response with angles of $0^{\circ}$ and $2^{\circ}$ almost coincide. The most significant change occurs with an angle of $8^{\circ}$.

A similar experiment was made with microphones without the front cavity, type B\&K 4145, without protection grid; Fig. 6 shows the results. As mentioned before, there is a geometrical difference between an LS1 microphone and the B\&K 4145; the former has a front cavity and the latter has not. It is known that this difference has a significant effect on the free-field sensitivity. ${ }^{17}$ The temporal positions of the echoes are the same as in the previous experiment, but the change in the amplitude and shape is much less pronounced. In fact, there is no significant change in the amplitude at any angle, although the shape of the echo seems to widen and split into two components at $8^{\circ}$ in the same way as observed with the B\&K 4160 microphones. Presumably the stronger backscattering of the B\&K 4160 microphones and the larger effect of tilting the microphone can be attributed to a focusing effect due to the front cavity.

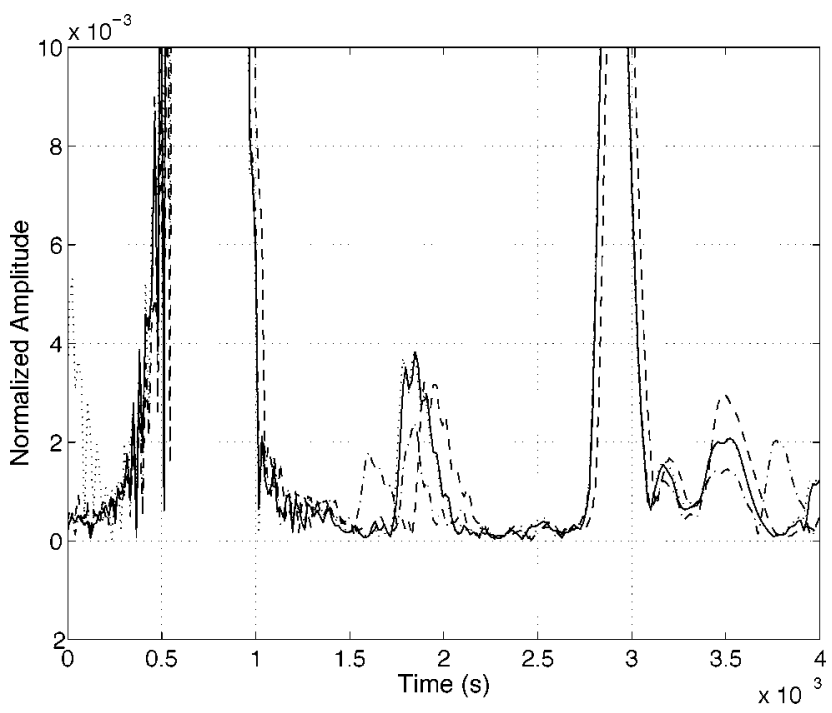

FIG. 5. The Hilbert envelope of the normalized impulse response corresponding to the electrical transfer impedance between two LS1 microphones (type B\&K 4160), one of which is tilted at different angles. - , $0^{\circ} ; \cdots, 2^{\circ} ;-$, $4^{\circ} ;-\cdot-, 8^{\circ}$. 


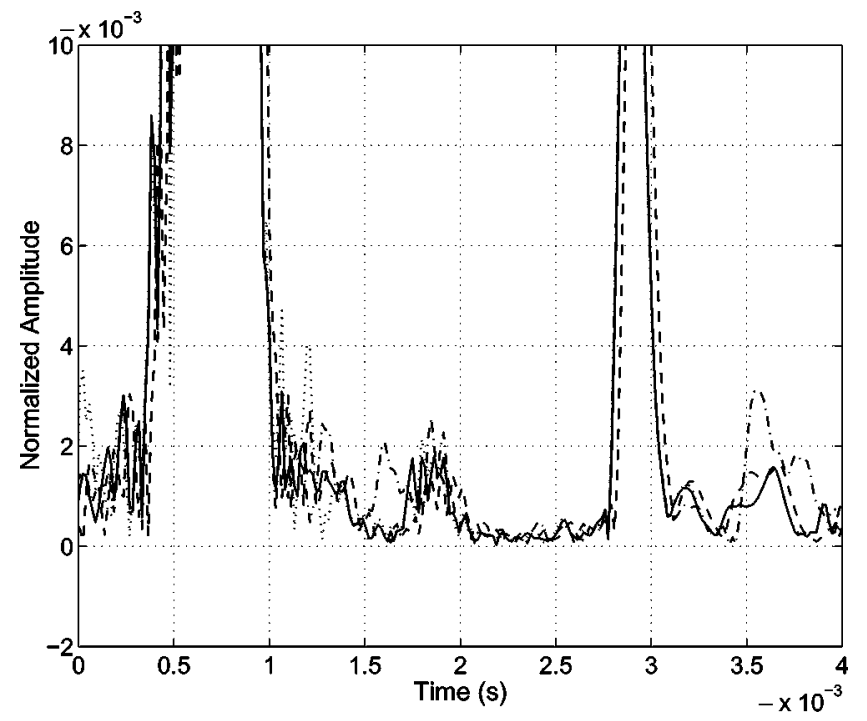

FIG. 6. The Hilbert envelope of the normalized impulse response corresponding to the electrical transfer impedance between two microphones of type $\mathrm{B} \& \mathrm{~K} 4145$ without a protection grid, one of which is tilted at different angles.,$- 0^{\circ} ; \cdots, 2^{\circ} ;-, 4^{\circ} ;-\cdot-, 8^{\circ}$.

\section{NUMERICAL RESULT}

To obtain a more realistic estimate of the amplitude of the secondary reflection than provided by approximating the microphones with spheres, a numerical solution of the scattered field is needed. This can be obtained by applying the Boundary Element Method (BEM).

First, the problem of obtaining an estimate of the factor $\left(p_{s, 2} / A\right)$ for frontal incidence is considered. For this purpose, an axisymmetric BEM formulation is used. ${ }^{13}$ A plane wave is incident on the body of a microphone mounted on a long rod. The rod has a length of $60 \mathrm{~cm}$ and is rounded at the end. The finite length introduces a small disturbance in the simulated results because of the reflections from the end, but it is expected that the amplitude of such a disturbance is small. The highest frequency in the calculations is $30 \mathrm{kHz}$. The size of the smallest element in the axisymmetric mesh is $2.5 \mathrm{~mm}$. Thus, there are at least four elements per wavelength at the highest calculation frequency.

It is well known that BEM solutions for exterior problems can be contaminated by spurious results associated with fictitious eigenfrequencies in the internal domain of the geometry. ${ }^{18}$ This is also known as the nonuniqueness problem. In the case at hand this problem can occur above about $10 \mathrm{kHz}$. The nonuniqueness problem has been avoided by adding a random CHIEF point, as described in Ref. 18 and further checked by calculating the condition numbers of the BEM matrices ${ }^{19}$ and by repeating calculations with small frequency shifts.

Figure 7 shows the modulus of the factor $\left(p_{s, 2} / A\right)$ for the microphones with and without a front cavity. The modulus has been determined from the BEM calculations and, for a comparison, also from the experimental results shown in Fig. 5. The BEM calculations are based on the ratio of the direct wave to the backscattered wave. The experimental results have been determined by time windowing the direct impulse response and the disturbance caused by the "stand-

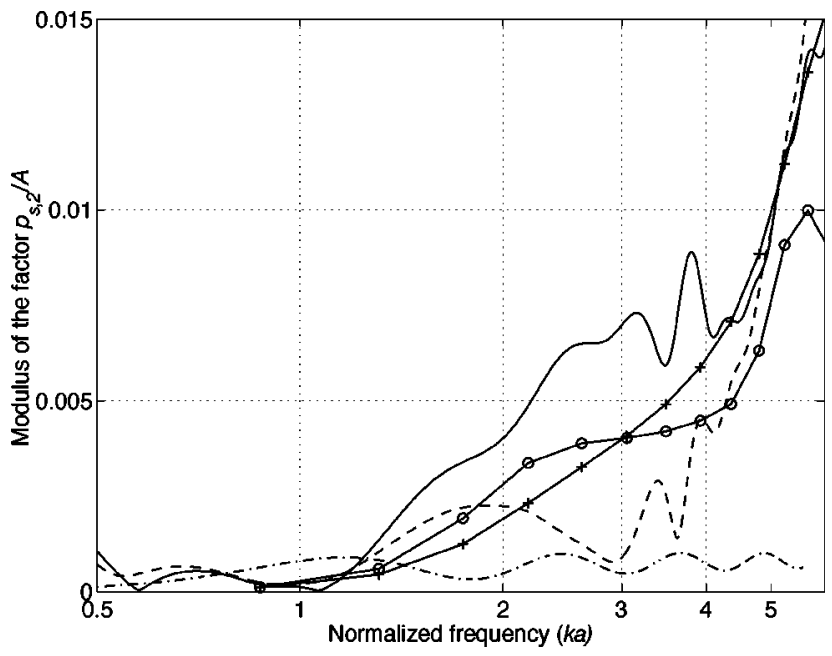

FIG. 7. The ratio of secondary reflection to direct sound at a distance of 200 $\mathrm{mm}$ between the objects. $-\mathrm{O}_{-}$, numerical results for LS1 microphones; -+- , numerical results for microphones of type B\&K 4145 without a protection grid; ---, spheres; - , experimental results for LS1 microphones (type B\&K 4160); -, experimental results for microphones of type B\&K 4145 without a protection grid.

ing wave" using the technique described in Ref. 11, and then transforming them to frequency responses by applying the Fourier transform, and eventually calculating the ratio of the disturbance to the direct wave. The case of the sphere is also presented.

It can be seen that the ratio is significantly larger for microphones than for spheres. It is also apparent that there are substantial differences between the backscattering of the two types of microphones. Whereas the calculated ratio for the microphone without the front cavity increases nearly monotonously with the frequency, there is a change in the slope from $k a$ values between 2.5 and 5 for the microphone with a front cavity. This more irregular behavior may be caused by the influence of the first radial resonance of the front cavity. The experimental results for the B\&K 4160 microphones appear to be in tolerable agreement with the numerical calculations, considering the fact that they are experimental second-order data determined with a very poor signal-tonoise ratio. The experimental results for the $\mathrm{B} \& \mathrm{~K} 4145 \mathrm{mi}-$ crophones are in poor agreement with the numerical results for $k a$ values between 2 and 5 .

Finally, it would be interesting to have an illustration of the sound field around the microphones. This can be determined by simulating two microphones in front of each other. The diaphragm displacement, $\eta$, of the transmitter microphone under uniform pressure conditions is

$$
\eta(r)=\left[\frac{J_{0}\left(k_{M} r\right)}{J_{0}\left(k_{M} a\right)}-1\right] \eta_{0},
$$

where $J_{0}$ is the Bessel function of zeroth order, $\eta_{0}$ is a constant that describes the amplitude of the movement, $k_{M}$ is the wave number of the diaphragm, $a$ is the radius of the diaphragm, and $r$ is the radial coordinate. Equation (14) is expected to represent the actual distribution fairly well. ${ }^{20}$ Thus, the simulated geometry consists of two microphones mounted on rods, facing each other. The same meshing parameters as in the previous calculations were used, but this 


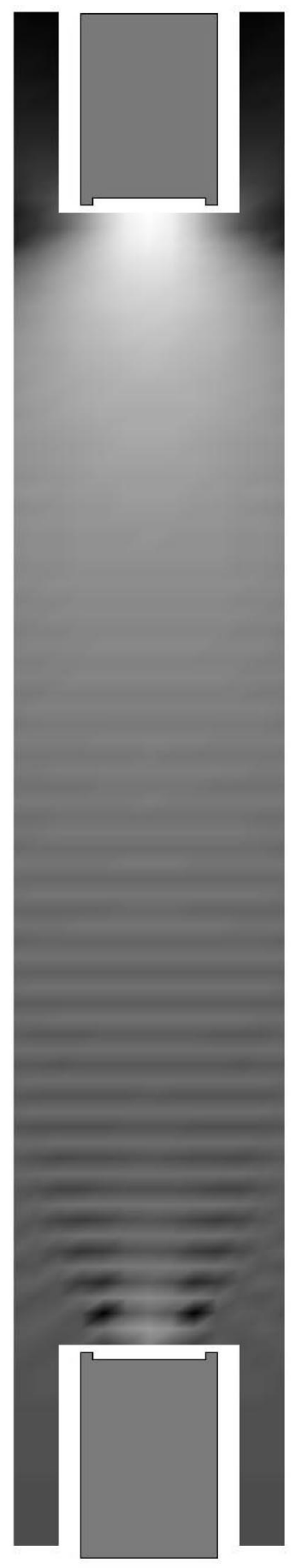

FIG. 8. The sound field between the two LS1 microphones, $20 \mathrm{~cm}$ apart in a free-field reciprocity setup at $20 \mathrm{kHz}$. The dark zones indicate minimum values, and the bright zones indicate the maximum values of the sound pressure.

time the sound pressure was calculated in a mesh around the microphones. Figure 8 shows the calculated sound field around for the LS1 microphones at $20 \mathrm{kHz}$. An interference pattern is apparent in front of the receiver microphone.

\section{DISCUSSION}

The term "standing wave" is somewhat misleading, and it would seem to be better to call the phenomenon an interference between the direct wave and the result of scattering by the two microphones. This interference cannot be eliminated by tilting the axis of one of the microphones a few degrees, as suggested; fairly large angles are required. However, this would introduce additional complications. For example, the acoustic center of a microphone, which is essential to know in free-field reciprocity calibration, ${ }^{1}$ depends on the angle of incidence.

The interference problem is larger for LS1 microphones, which have a front cavity, than for microphones without a front cavity, such as B\&K 4145 without a protecting grid, in agreement with the fact that the latter have a more uniform directional behavior as a function of frequency than microphones with a front cavity. ${ }^{17}$

\section{CONCLUSIONS}

The interference between the two microphones in a freefield reciprocity calibration has been analyzed in some detail. The phenomenon is an interference effect due to multiple backscattering from the bodies of the transducers rather than a "standing wave" between the diaphragms. Therefore, tilting the axis of one of the microphones cannot eliminate the phenomenon. Time-selective techniques seem to be more appropriate.

\section{ACKNOWLEDGMENT}

The authors would like to thank Vicente Cutanda for his constructive criticism.

${ }^{1}$ IEC 61094-3 (1995-11), "Measurement microphone Part 3: Primary method for free-field calibration of laboratory standard microphones by the reciprocity technique," 1995.

${ }^{2}$ W. R. MacLean, "Absolute measurement of sound without a primary standard," J. Acoust. Soc. Am. 12, 140-146 (1940).

${ }^{3}$ W. Wathen-Dunn, "On the reciprocity free field calibration of microphones," J. Acoust. Soc. Am. 21, 542-546 (1949).

${ }^{4}$ I. Rudnick and M. N. Stein, "Reciprocity free field calibration of microphones to $100 \mathrm{Kc}$ in air," J. Acoust. Soc. Am. 20, 818-825 (1948).

${ }^{5}$ E. W. Burnett and V. Nedzelnitsky, "Free-field reciprocity calibration of microphones,” J. Res. Natl. Bur. Stand. 92, 129-151 (1987).

${ }^{6}$ D. L. H. Gibbings and A. V. Gibson, "Free-field reciprocity calibration of capacitor microphones at frequencies from $19.95 \mathrm{kHz}$ to $316.2 \mathrm{~Hz}$," Metrologia 20, 85-94 (1984).

${ }^{7}$ J.-N. Durocher, "Etalonnage des microphones à condensateur en champ libre," J. d'Acoust. 2, 431-436 (1989).

${ }^{8}$ K. Rasmussen and E. Sanderman Olsen, "Intercomparison on free-field calibration of microphones," The Acoustics Laboratory, Technical University of Denmark, Report PL-07, 1993.

${ }^{9}$ L. L. Foldy and H. Primakoff, "A general theory of passive linear electroacoustic transducers and the electroacoustic reciprocity theorem. I," J. Acoust. Soc. Am. 17, 109-120 (1945).

${ }^{10}$ R. J. Bobber, "Diffraction constants of transducers," J. Acoust. Soc. Am. 37, 591-595 (1965)

${ }^{11}$ S. Barrera-Figueroa, K. Rasmussen, and F. Jacobsen, "A time-selective technique for free-field reciprocity calibration," J. Acoust. Soc. Am. 114, 1467-1476 (2003).

${ }^{12}$ I. Ihlárova and F. Jacobsen, "An approximate method of modelling scattering by composite bodies," J. Sound Vib. 262, 1235-1241 (2003).

${ }^{13}$ P. M. Juhl, "A numerical investigation of standard condenser microphones," J. Sound Vib. 177, 433-446 (1994).

${ }^{14}$ P. M. Morse and K. U. Ingard, Theoretical Acoustics (McGraw-Hill, New York, 1968/1984). See Sec. 8.2.

${ }^{15}$ F. Ingerslev, O. J. Pedersen, P. K. Møller, and J. Kristensen, "New rooms for acoustic measurements at the Danish Technical University," Acustica 19, 187-199 (1967/68).

${ }^{16}$ A. D. Pierce, Acoustics. An Introduction to Its Physical Principles and 
Applications (American Institute of Physics, New York, 1989). See Sec. 9.1.

${ }^{17}$ Brüel \& Kjær technical documentation, "Condenser microphones data handbook," Nærum, 1982.

${ }^{18} \mathrm{H}$. A. Schenck, "Improved integral formulation for acoustic radiation problems," J. Acoust. Soc. Am. 44, 41-58 (1968).
${ }^{19}$ P. M. Juhl, F. Jacobsen, V. Cutanda, and S. Quirós Alpera, "On the nonuniqueness problem in a 2-D half-space BEM formulation," Proceedings of the 9th International Congress on Sound and Vibration, Orlando, FL, 2002 (CD-ROM).

${ }^{20}$ A. J. Zuckerwar, "Theoretical response of condenser microphones," J. Acoust. Soc. Am. 64, 1278-1285 (1978). 